\title{
REA ja oder nein?
}

Bernhard Gurtner

Korrespondenz:

Dr. med. Bernhard Gurtner

Eggstrasse 76

CH-8620 Wetzikon
«Hat der Patient im Zimmer A 37 REA oder nein?» «Würden Sie bitte noch die REA unterschreiben?» Welcher Spitalarzt kennt diese Fragen nicht! [1] Und wie viele Kliniken gibt es, in denen das Pflegepersonal schon am Aufnahmetag bei allen Neueintretenden das Feld REA ja/nein im Krankendossier abhaken möchte, damit bei einem allfälligen Zwischenfall in der ersten Spitalnacht kein unnötiger Alarm ausgelöst wird? Dieser Wunsch ist ebenso verständlich wie gefährlich, weil er zu improvisierten und schlecht kommunizierten Entscheidungen verleitet und trotz beschwichtigender Beteuerungen die Gefahr in sich birgt, dass die ärztliche und pflegerische Zuwendung bei nicht «reanimationswürdigen» Patienten gedrosselt wird.

Es gibt eine Mehrzahl von Patientinnen und Patienten, bei denen allen Beteiligten klar ist, dass sie reanimiert würden, auch ohne KG-Eintrag. Bei einer zweiten Gruppe ist es offensichtlich, dass sie in ihrem Leben und Leiden einen terminalen Zustand erreicht haben, weshalb eine REA-Diskussion nur unbegründete Hoffnungen und zusätzliche Ängste auslösen würde. In den britischen Richtlinien vom Oktober 2007 [2] gilt denn auch:

«When a clinical decision is made that CPR should not be attempted, because it will not be successful, and the patient has not expressed a wish to discuss $\mathrm{CPR}$, it is not necessary or appropriate to initiate discussion with the patient to explore their wishes regarding CPR.»

Im zur Vernehmlassung bis 15. September 2008 im Internet abrufbaren Richtlinienentwurf «Reanimationsentscheidungen» der SAMW [3] findet sich hingegen die strikte Forderung:

«In Situationen, in welchen eine Reanimation medizinisch nicht indiziert ist, muss der behandelnde Arzt das Gespräch mit dem Patienten suchen. Dieses soll mit der notwendigen Sorgfalt und Empathie geführt werden.»

Das besagt, dass allen Neueintretenden, bei denen aus offensichtlichen Gründen REA nein angekreuzt wurde, aufwühlende Gespräche aufgedrängt werden müssten, die nicht nur sie selbst, sondern oft auch das betreuende Team überfordern würden. Ist das die Meinung der Subkommission, die immerhin davor warnt, Sterbende mit einem Gespräch über Reanimation zu belasten?

Entscheidungen im eigentlichen Wortsinn sind nur dann zu treffen, wenn die medizinische Situation ungewiss ist oder wenn die Kranken selbst, ihre Angehörigen, das betreuende Pflegeteam und die verantwortlichen Ärztinnen oder Ärzte unterschiedliche Meinungen und Wünsche äussern. Es braucht in diesen schwierigen Fällen eine sorgfältige Güterabwägung und vermittelnde Diskussionen mit allen Beteiligten. Diese Aspekte werden im Richtlinienentwurf ausführlich dargelegt.

Die raschen Einträge REA ja / nein sind nur für die mit grosser Wahrscheinlichkeit profitierenden oder ebenso eindeutig chancenlosen Kranken vertretbar, sie bringen aber für das Pflegepersonal eine entlastende Klärung der Situation. In fraglichen Fällen jedoch darf ein DNAR-Vermerk nie mit einem raschen Ankreuzen erfolgen, er muss begründet und vom Verantwortlichen unterzeichnet werden. Das haben wir alten Kaderärzte öfters nicht getan [4], zweifellos eine ungerechtfertigte Anmassung. In diesem Sinne sind neue Entscheidungshilfen begrüssenswert. Der praktische Nutzen des umfangreichen Papiers könnte noch verbessert werden durch eine stichwortartige Auflistung der wichtigsten Merkpunkte und ein Decisionmaking-Framework (siehe [2], Seite 22) nach dem englischen Vorbild.

\section{Literatur}

1 Gerber A, Salathé M. Reanimationsentscheidungen. Schweiz Ärztezeitung. 2008;89(23):1014.

2 Decisions relating to cardiopulmonary resuscitation. A joint statement from the British Medical Association, the Resuscitation Council (UK) and the Royal College of Nursing. October 2007. www.bma.org.uk/ap.nsf/Content/CPRDecisions07.

3 www.samw.ch $\rightarrow$ Ethik $\rightarrow$ Richtlinien $\rightarrow$ Reanimationsentscheidungen

4 Gurtner B. REAlistische Betrachtungen. Schweiz Ärztezeitung. 2006;87(29/30):1348-9. 